\title{
Dll4 Blockade Promotes Angiogenesis in Nonhealing Wounds of Sox7-Deficient Mice
}

\author{
Jee Myung Yang, ${ }^{1, \dagger}$ Junghwa Ryu, ${ }^{2, \dagger}$ Injune Kim, ${ }^{1, *}$ \\ Hak Chang, ${ }^{3, *}$ and II-Kug Kim ${ }^{2, *}$ \\ ${ }^{1}$ Graduate School of Medical Science and Engineering, Korea Advanced Institute of Science and Technology, Daejeon, \\ Republic of Korea. \\ ${ }^{2}$ Department of Plastic and Reconstructive Surgery, Yeungnam University College of Medicine, Daegu, Republic of Korea. \\ ${ }^{3}$ Department of Plastic and Reconstructive Surgery, Seoul National University College of Medicine, Seoul, Republic of Korea. \\ ${ }^{\dagger}$ Co-first authors.
}

Objective: This study aimed to elucidate the role of the proangiogenic transcription factors Sox7 and Sox17 in the wound healing process and investigate the therapeutic potential of Dll4 blockade, which is an upstream regulator of Sox17, for the treatment of nonhealing wounds.

Approach: After generating a full-thickness skin defect wound model of endothelial Sox7- and/or Sox17-deficient mice, we measured the wound healing rates and performed histological analysis. The effects of an anti-Dll4 antibody on wound angiogenesis in Sox7-deficient mice and $d b / d b$ diabetic mice were assessed.

Results: Sox7 and/or Sox17 deletion delayed wound healing. Moreover, the loss of Sox7 and Sox17 inhibited wound angiogenesis, without affecting the expression of the other. Of interest, after anti-Dll4 antibody treatment, Sox17 levels were increased and the suppression of angiogenesis was alleviated in Sox7-deficient mice and $d b / d b$ diabetic mice. Consequently, Dll4 blockade effectively recovered the observed delay in wound healing.

Innovation: The proangiogenic role of Sox 7 and Sox17 in wound angiogenesis was addressed and effective treatment of nonhealing wounds by Dll4 blockade was suggested.

Conclusion: This study revealed the proangiogenic role of the transcription factors Sox7 and Sox17 in wound angiogenesis. Furthermore, we suggest a novel method for treating nonhealing wounds by particularly targeting the Dll4-Sox17 axis.

Keywords: wound healing, angiogenesis, transcription factor, Sox7, Sox17, delta-like ligand 4

\section{INTRODUCTION}

ANGIOGENESIS Is A critical process for wound healing, as it involves the growth of capillary sprouts to granulation tissue. ${ }^{1-3}$ Impaired angiogenesis and nonhealing wounds in numerous clinical conditions. ${ }^{2,4-6}$ Although preis considered a common pathophysiological mechanism underlying chronic vious studies on wound angiogenesis have examined various angiogenic cytokines such as vascular endothelial growth factor (VEGF), fibroblast growth factor, epidermal growth factor, transforming growth factor- $\beta$, and angiopoietin, along with the relevant signaling pathways, no method for effectively promoting angiogenesis in
(C) Jee Myung Yang, et al. 2019; Published by Mary Ann Liebert, Inc. This Open Access article is distributed under the terms of the Creative Commons Attribution Noncommercial License (http://creative commons.org/licenses/by-nc/4.0/) which permits any noncommercial use, distribution, and reproduction in any medium, provided the original author(s) and the source are cited.

\section{a}

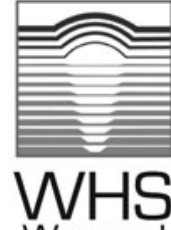

Wound

Healing

Society

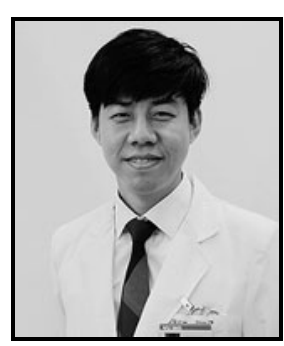

II-Kug Kim, MD, PhD

Submitted for publication May 10, 2019. Accepted in revised form October 29, 2019.

*Correspondence: II-Kug Kim, MD, PhD, Department of Plastic and Reconstructive Surgery, Yeungnam University College of Medicine, Daegu 42415, Republic of Korea (e-mail: curingyou@yu.ac.kr)

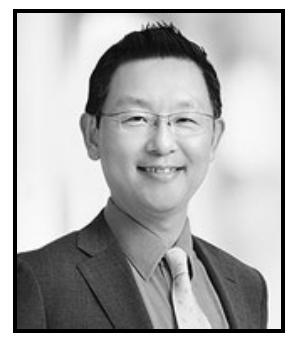

Hak Chang, MD, PhD

Department of Plastic and Reconstructive Surgery, Seoul National University College of Medicine, Seoul 03080, Republic of Korea (e-mail: hchang@snu.ac.kr)

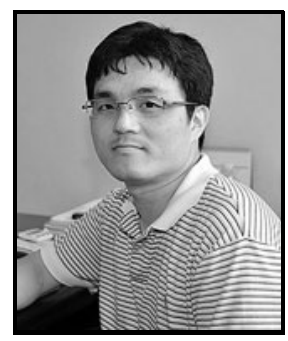

Injune Kim, PhD

Graduate School of Medical Science and Engineering, Korea Advanced Institute of Science and Technology, Daejeon 34141, Republic of Korea (e-mail: injunek@kaist.ac.kr). 
nonhealing wounds has yet been proposed. ${ }^{2,7}$ Thus, a deeper understanding of angiogenic regulation during the wound healing process is required.

Recently, studies on developmental and tumor angiogenesis have focused on the proangiogenic transcription factors Sox7 and Sox17 and their related signaling pathways. ${ }^{8-11}$ Both transcription factors display endothelial cell (EC)-specific expression in developing and tumor vessels and their deletion inhibits angiogenesis. Accordingly, studies on the role of Sox7 and Sox17 in angiogenesis will improve the understanding of the transcriptional regulation of angiogenesis not only in developmental or tumor vessels but also in other types of angiogenesis. However, their expression patterns and roles in wound angiogenesis remain unknown, which were investigated in this study.

To target angiogenic pathways involving Sox7 and Sox17 in wound tissues, their upstream regulator must be evaluated. Dll4-Notch signaling is induced by VEGF as a negative feedback regulator of angiogenesis, ${ }^{12}$ negatively regulating Sox17 in several contexts; moreover, it serves as a candidate upstream regulator for Sox7 and Sox17 in wound angiogenesis. ${ }^{9,10}$ Therefore, this study aimed to investigate whether Dll4 blockade promotes angiogenesis and improves wound healing in nonhealing wounds of Sox7-deficient mice.

\section{CLINICAL PROBLEM ADDRESSED}

Numerous individuals worldwide have chronic wounds. In the United States alone, $\$ 25$ billion is annually spent on treating chronic wounds. Various treatment methods have been suggested; however, numerous patients do not present improvement, and advanced treatments are expensive. In treating chronic wounds with impaired angiogenesis, two broad clinical problems exist. First, diagnostic markers to quantify angiogenesis are lacking. Assessment of the distribution of blood vessels exclusively through histological analysis is not sufficient because it is difficult to determine whether the blood vessels mediate active angiogenesis or are dysfunctional. This study examined the expression of the proangiogenic transcription factors Sox7 and Sox17 in wound tissues for their use as markers of angiogenesis levels. Second, a stable antibody potentially serving as a therapeutic agent for inducing angiogenesis in tissues with impaired angiogenesis is lacking. We examined the role of the Dll4-Sox17 axis in wound healing and confirmed whether antiDll4 antibody induces angiogenesis in nonhealing wounds with impaired angiogenesis.

\section{MATERIALS AND METHODS \\ Mice}

Cdh5(BAC)-CreER ${ }^{\mathrm{T} 2}$, So $x 7^{\mathrm{mCh} /+}$, Sox $17^{\mathrm{GFP} /+}$, $\operatorname{Sox} 7^{\mathrm{fl} / \mathrm{fl}}, \operatorname{Sox} 17^{\mathrm{f} / \mathrm{fl}}$, and $\operatorname{Sox} 7^{\mathrm{fl} / \mathrm{fl}} / \operatorname{Sox} 17^{\mathrm{fl} / \mathrm{fl}}$ mice were housed in a pathogen-free animal facility. To excise Sox7, Sox17, and Sox7/Sox17 in ECs, the Cdh5 (BAC)-CreER ${ }^{\mathrm{T} 2}$ driver line was bred with $\operatorname{Sox} 7^{\mathrm{f} / \mathrm{fl}}$, $\operatorname{Sox} 17^{\mathrm{f} / / \mathrm{fl}}$, and $\operatorname{Sox} 7^{\mathrm{f} / \mathrm{fl}} / \operatorname{Sox} 17^{\mathrm{f} / \mathrm{fl}}$ mice, respectively. Cre-ER ${ }^{\mathrm{T} 2}$-negative but flox/flox-positive mice among the littermates were used as the control for each experiment. For Cre activation, $20 \mathrm{mg} / \mathrm{kg}$ tamoxifen (Sigma, St. Louis, MO) was intraperitoneally administered to mice four times with intervals of 3 days, beginning at 10 to 12 weeks of age. Thereafter, full-thickness skin defects were generated at the flank area. Genetically diabetic mice (db/db; BKS.Cg-Dock $\left.7^{\mathrm{m}}+/+\mathrm{Lepr}^{\mathrm{db}} / \mathrm{J}\right)$ 10-12 weeks of age were purchased from Jackson Laboratory (Bar Harbor, ME) for the diabetic wound healing experiment. Serum glucose levels of $>300 \mathrm{mg} / \mathrm{dL}$ confirmed the diabetic phenotype in $d b / d b$ mice. We used male mice only in this study to obtain consistent results. To block Dll4, $5 \mathrm{mg} / \mathrm{kg}$ of the anti-Dll4 antibody (clone 26.82, US7803377 B2) or an equal amount of control antibody was intraperitoneally administered to Sox7-deficient mice at 1,4 , and 7 days after surgery. Furthermore, $5 \mathrm{mg} / \mathrm{kg}$ of the anti-Dll4 antibody was injected into diabetic $d b / d b$ mice at 7,10 , and 13 days after surgery. Mice were anesthetized with $80 \mathrm{mg} / \mathrm{kg}$ ketamine and $12 \mathrm{mg} / \mathrm{kg}$ xylazine, and normal skin or wound tissue specimens were harvested for further analyses. All animal experiments were approved by the Animal Care Committee of Korea Advanced Institute of Science and Technology (KA2018-02).

\section{Mouse wound splinting model}

Mice were anesthetized and the hair on their flank area was removed. A full-thickness skin defect was induced on the back of each mouse, using a $7 \mathrm{~mm}$ biopsy punch (Miltex Instrument, York, PA). Thereafter, donut-like silicone splints (inner diameter $=10 \mathrm{~mm}$, outer diameter $=20 \mathrm{~mm}$ ) fabricated from $0.5 \mathrm{~mm}$ thick silicone sheets (Grace BioLabs, Bend, OR) were applied to the skin $1 \mathrm{~mm}$ from the wound perimeter and affixed with eight interrupted stitches made using 5-0 black silk sutures. The wounds were completely covered with Tegaderm (3M) sterile transparent dressing and a self-adhering elastic bandage.

\section{Histological analyses}

For immunofluorescence staining, wound tissues were fixed overnight in $4 \%$ paraformaldehyde, dehydrated in $20 \%$ sucrose solution overnight, and 
embedded in tissue-freezing medium (Leica, Wetzlar, Germany). Frozen blocks were cut into $20 \mu \mathrm{m}$ thick sections. Tissue sections were blocked in PBST $(0.2 \%$ Tween-20 in PBS) with $5 \%$ goat or donkey serum and incubated with the following primary antibodies: anti-CD31 (hamster, 2H8; Millipore, Billerica, MA; or rat, MEC13.3; BD Biosciences, Franklin Lakes, NJ), anti-Sox7 (goat; R\&D Systems, Minneapolis, MN), anti-Sox17 (goat; R\&D Systems), and anti-VEGFR2 (goat; R\&D Systems) antibodies. After several washes, the sections were incubated with the following secondary antibodies: Alexa Fluor 488- or Alexa Fluor 596conjugated anti-hamster IgG, Alexa Fluor 488- or Alexa Fluor 596-conjugated anti-rat IgG, or Alexa Fluor 488- or Alexa Fluor 596-conjugated anti-goat IgG (Jackson ImmunoResearch, West Grove, PA) antibodies. Nuclei were stained with DAPI (Invitrogen, Carlsbad, CA). The samples were mounted with fluorescent mounting medium (DAKO, Glostrup, Denmark), and immunofluorescence images were obtained using a confocal microscope (LSM800; Zeiss, Jena, Germany).

\section{Assessment of hypoxia and vascular perfusion}

To analyze the hypoxic areas of wounds, $60 \mathrm{mg} / \mathrm{kg}$ pimonidazole hydrochloride (Natural Pharmacia International, Burlington, MA) was intraperitoneally administered $60 \mathrm{~min}$ before the wound tissues were harvested. Wound tissues were stained with fluorescein isothiocyanate (FITC)-conjugated antihypoxyprobe antibody. Hypoxic areas were determined as percentages of the FITC-positive area per random $0.54 \mathrm{~mm}^{2}$ region. For vascular perfusion, $100 \mu \mathrm{L}$ of DyLight 488-conjugated tomato lectin $(1 \mathrm{mg} / \mathrm{mL}$, Vector laboratory) was intravenously administered $30 \mathrm{~min}$ before euthanasia. The vascular perfusion area was determined as a percentage of the lectin-positive area divided by the CD31positive area. Mice were anesthetized and intracardially perfused with PBS to eliminate circulating pimonidazole or lectin. To minimize local variation, two different images from each section were obtained and mean pimonidazole or lectin levels were measured. All signal intensities were measured using ImageJ software (National Institutes of Health, Bethesda, MD). The threshold was fixed in the same level.

\section{Densitometric analyses}

The vessel area was determined as the CD31positive area per random $0.54 \mathrm{~mm}^{2}$ region. Sox7, Sox17, Sox7-mCherry, Sox17-GFP, and VEGFR2 expression levels were determined as percentages of the corresponding fluorescence-positive areas per CD31-positive blood vessel. The densities of immunofluorescence signals were quantified using ImageJ software. To minimize local variation, we obtained two different images from each section and determined the mean expression levels.

\section{Statistical analysis}

Data are presented as mean \pm standard deviation values, unless otherwise indicated. Statistical differences were determined by performing the Mann-Whitney $U$-test for two groups or the Kruskal-Wallis test followed by the rank-based Tukey's honest significant difference test for more than two groups. Statistical analyses were performed using SPSS version 22 software (SPSS, Inc., Chicago, IL). $p<0.05$ was considered significant.

\section{RESULTS}

\section{Sox7 and Sox17 are upregulated in ECs during wound healing}

Because Sox7 and Sox17 are upregulated in ECs during development and tumor angiogenesis, we investigated their expression in tissues during normal wound healing. Normal skin and fullthickness skin-defect wounds were harvested from Sox $7^{\mathrm{mCh} /+}$ and $S o x 17^{\mathrm{GFP} /+}$ mice at 3,6 , and 10 days after surgery. mCherry and GFP fluorescence were observed in Sox $7^{m C h /+}$ and Sox $17^{G F P /+}$ mice to evaluate Sox7 and Sox17 expression. CD31 was used as a marker of ECs in the normal skin and wound tissue. Sox7-mCherry and Sox17-GFP were downregulated in normal skin in comparison with wound tissue. Sox7-mCherry signal intensity in ECs gradually increased until day 6 and slightly decreased by day 10 after surgery (Fig. 1A; day 6, $66.1 \pm 3.5$; day $10,61.3 \pm 4.2$ ). However, no significant difference in Sox7-mCherry signals was observed between days 6 and 10. Because Sox 17 is a closely related homolog of Sox7, we also assessed endothelial Sox17 expression in the normal skin and wound tissues of Sox $17^{G F P /+}$ mice. Sox17-GFP signals in ECs peaked on day 6 after surgery (Fig. 1B; day 6, 61.1 \pm 6.4 ; day $10,56.7 \pm 3.8$ ). These results indicate that ECs express Sox 7 and Sox17 at high levels during wound healing, suggesting that they play a role in angiogenesis during this process.

\section{Sox7 and/or Sox17 deletion delay wound healing and inhibit angiogenesis}

To investigate the role of Sox7 and Sox17 during wound healing, we generated full-thickness skin defects in the mouse flank and determined the wound closure rate in EC-specific Sox7-, Sox17-, or Sox7/Sox17 (double) knockout mice ( $n=10$ per group). After surgery, we observed the wounds by 
digital imaging at 3,6 , and 10 days postoperation; the mice were then killed on day 10 for immunohistological analysis. The wounds of control mice were completely closed by day 10 (Fig. 2A). In contrast, the wounds of Sox7, Sox17, or Sox7/ Sox17 double-deficient mice did not completely heal on day 10 (Fig. 2A). In control mice, the size of the full-thickness skin defect rapidly decreased; in particular, the lesions were reduced to $20 \%$ of the initial size after 3 days and completely healed within 10 days (Fig. 2B). However, in Sox7- or Sox17-deficient mice, wound healing was delayed, and lesion sizes decreased to $40 \%$ of their initial sizes on day 3, with incomplete closure on day 10 (Fig. 2B). Furthermore, Sox7/Sox17 doubledeletion mice exhibited delayed healing with the lesion size decreased to only $70 \%$ of the initial size on day 3 , which was further delayed in comparison with that observed in Sox7- or Sox17-deletion mice; again, the wounds did not completely heal by day 10 (Fig. 2B). These results suggest that endothelial Sox7 and Sox 17 are required for wound healing.

Next, we performed immunofluorescence analysis for CD31 to investigate whether delayed wound healing in Sox7, Sox17, or Sox7/Sox 17 double-deficient mice was associated with decreased angiogenesis in wounds. Vascularity in the granulation area was examined 10 days after surgery. Sox7 and Sox17 were upregulated and surface wounds completely healed in control mice on day 10; however, the wound persisted in the other groups at this time point. Thus, we analyzed the histological differences between the groups on day 10 after surgery to investigate factors affecting this phenotype. CD31 staining indicated a significant reduction in the blood vessel density in Sox7- or Sox17-deficient mice in comparison with control mice (Fig. 2C). Moreover, the vessel area in Sox7/ Sox17 double-deficient mice was markedly decreased in comparison with that in not only control mice $(p<0.001)$, but also in Sox7- or Sox17deficient mice $(p<0.01)$. These data indicate that Sox 7 and Sox 17 deletions impaired vascularity in wound tissue and that these effects are not redundant.

\section{Loss of Sox7 or Sox17 does not affect the expression of each other in wound tissues}

Depending on the context, Sox7 and Sox17 may affect the expression of each other. ${ }^{8,9,13}$ As Sox7/ Sox 17 double deletion markedly influenced vascularity in the wound tissue in comparison with the individual deletion of either protein, and each transcription factor was predicted to play an independent role. To confirm this, their respective effects on each other were investigated. In the wounds of control mice, Sox7 was expressed in $>20 \%$ of vessels but not in Sox7- and Sox7/Sox 17deficient mice (Fig. 3A). In the wounds of Sox 17deficient mice, Sox 7 was expressed in $16 \%$ of vessels

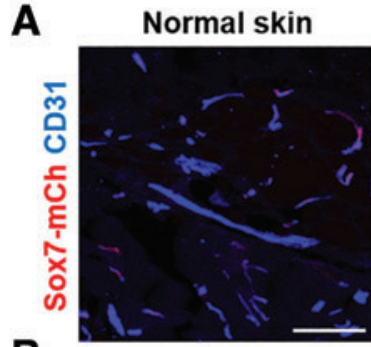

B
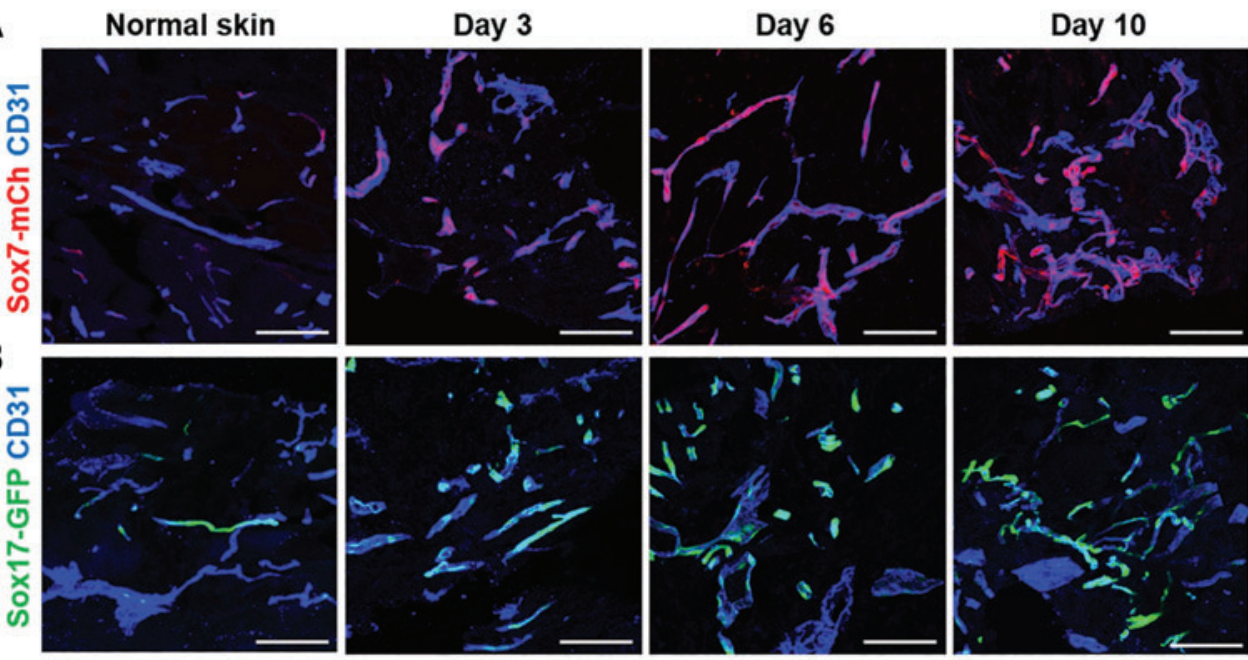

Scale bar: $100 \mu \mathrm{m}$

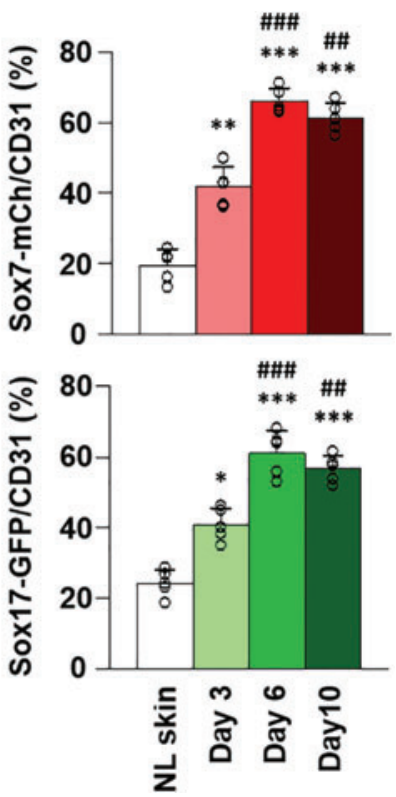

Figure 1. Endothelial-specific expression of Sox7 and Sox17 in wound tissues. (A) Sox7-mCherry fluorescence and signal quantification in normal skin and full-thickness skin defect wound on days 3,6, and 10. (B) Sox17-GFP fluorescence and signal quantification in normal skin and full-thickness skin defect wounds on days 3,6 , and 10 . Values are presented as the mean $\pm \operatorname{SD}(n=5) .{ }^{*} p<0.05 ;{ }^{* *} p<0.01 ;{ }^{* *} p<0.001$ versus normal skin; ${ }^{\# \# \#} p<0.01$ versus day 3 ; $\# \#<0.001$ versus day 3 . SD, standard deviation. Color images are available online. 

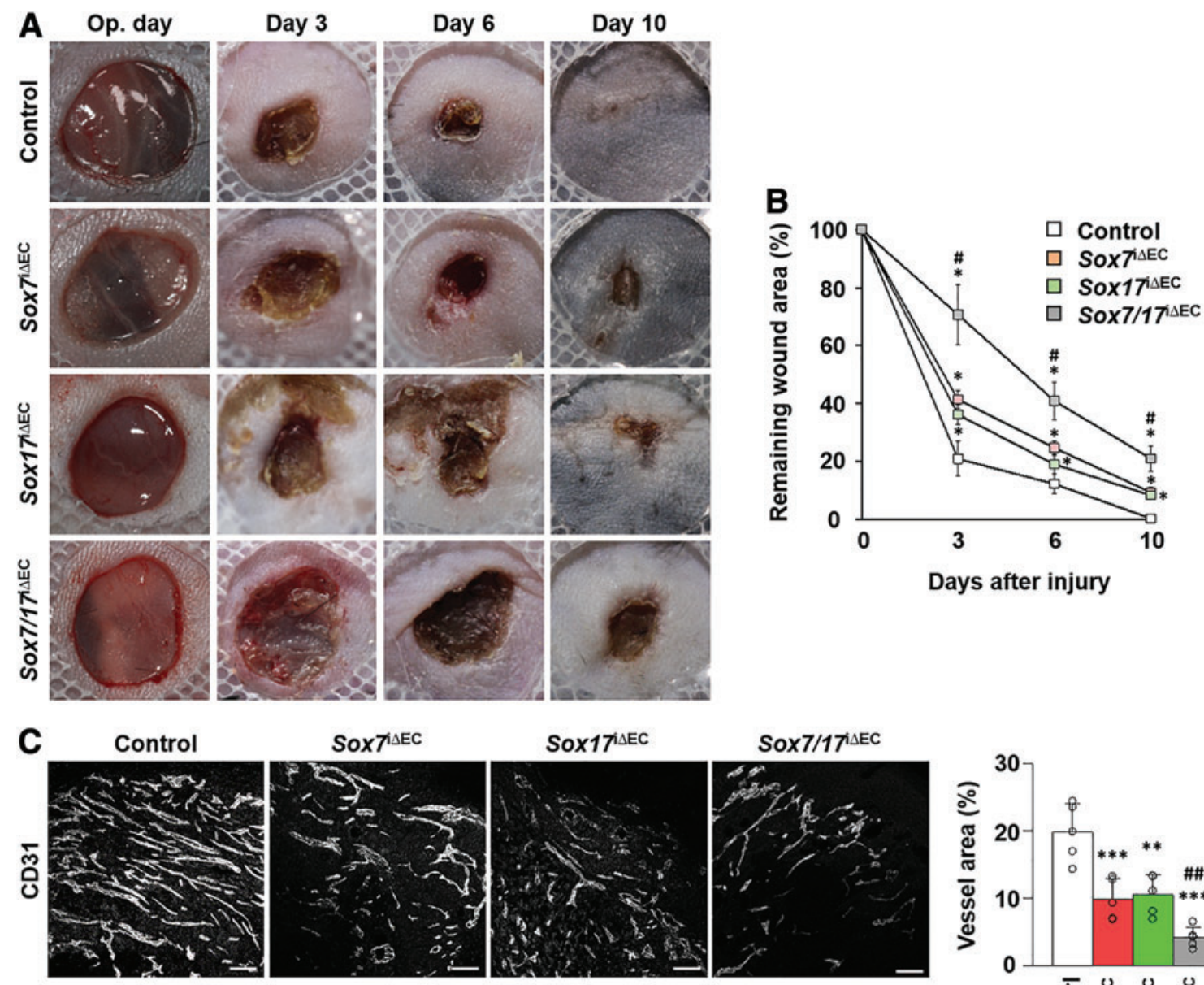

Scale bar: $100 \mu \mathrm{m}$

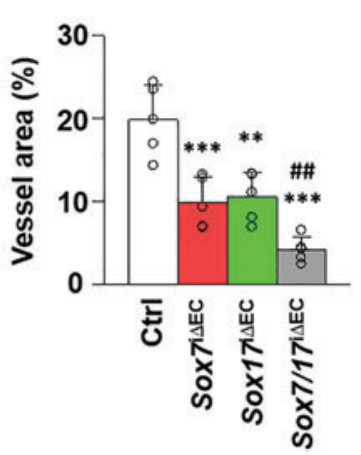

Figure 2. Sox7 and/or Sox17 deletion delays wound healing and inhibits wound angiogenesis. (A) Representative images of wounds at different time points. (B) Quantification of wound areas. (C) CD31-positive vessels in wound tissues. Values are presented as mean $\pm \operatorname{SD}(n=5) .{ }^{*} p<0.05 ;{ }^{* *} p<0.01$; ${ }^{* * *} p<0.001$; ${ }^{\#} p<0.05$ versus Sox7-KO; ${ }^{\# \#} p<0.05$ versus Sox7-KO. Color images are available online.

with no significant difference from control mice (Fig. 3A). Sox17 was expressed in $21 \%$ and $14 \%$ of vessels in the wounds of control and Sox7-deficient mice, respectively, and this difference was not significant (Fig. 3B). Sox17 was not expressed in the vessels of the wounds in Sox17 and Sox7/Sox 17 double-deficient mice (Fig. 3B). These results show that Sox 7 and Sox17 do not interfere with the expression of each other during wound angiogenesis; these results are concurrent with the observation that Sox7/Sox17 double deletion induces a more severe wound vascularity phenotype than single Sox 7 or Sox17 deletion.

\section{Sox7 and/or Sox17 deletions downregulate VEGFR2 and promote hypoxia}

To understand the mechanism underlying angiogenic impairment during wound healing caused by the deletion of Sox7 and/or Sox17, VEGFR2 expression and tissue hypoxia were examined. In the wounds of control mice with active angiogenesis, VEGFR2 was expressed in up to $60 \%$ of vessels (Fig. 3C). However, in the wound vessels of Sox 7- or Sox17-deficient mice, VEGFR2 expression was estimated up to $25-28 \%$, whereas in the wound vessels of Sox7/Sox17 double-deficient mice, VEGFR2 expression approached $11 \%$, indicating substantially reduced levels compared with those in control mice (Fig. 3C). These data suggest that VEGFR2 functions downstream of Sox7 and Sox17 during wound angiogenesis.

To investigate the microenvironment of the wound tissue, a hypoxyprobe (pimonidazole hydrochloride) was used to estimate hypoxia. In the wound tissue of control mice, estimated hypoxia was $13 \%$, whereas this value was $27-28 \%$ in Sox 7 - 
A

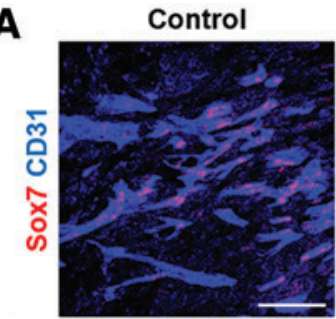

B
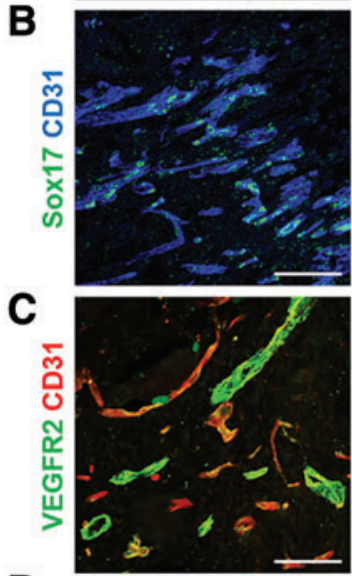

D

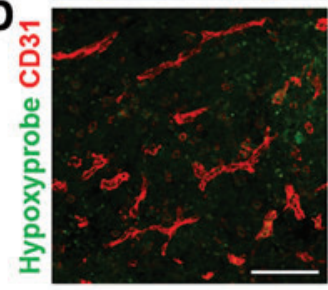

SOX ${ }^{\triangle A E C}$
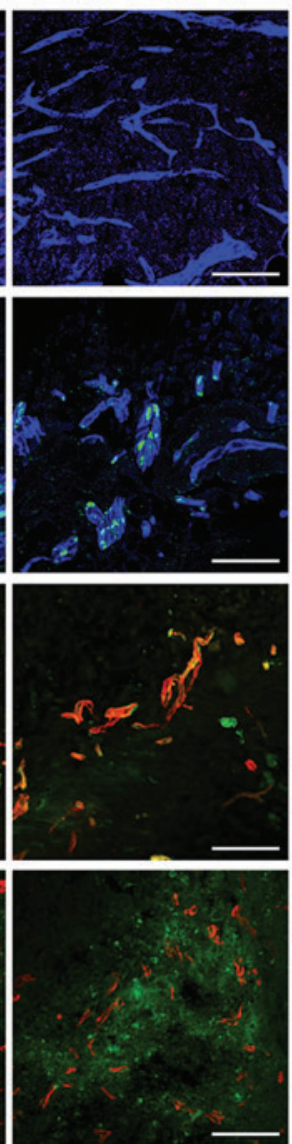

SOX17 $\triangle E C$
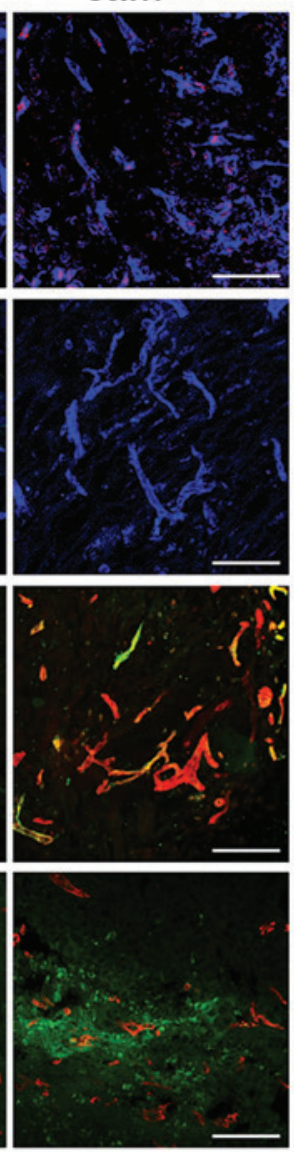

SOX7/17ISEC
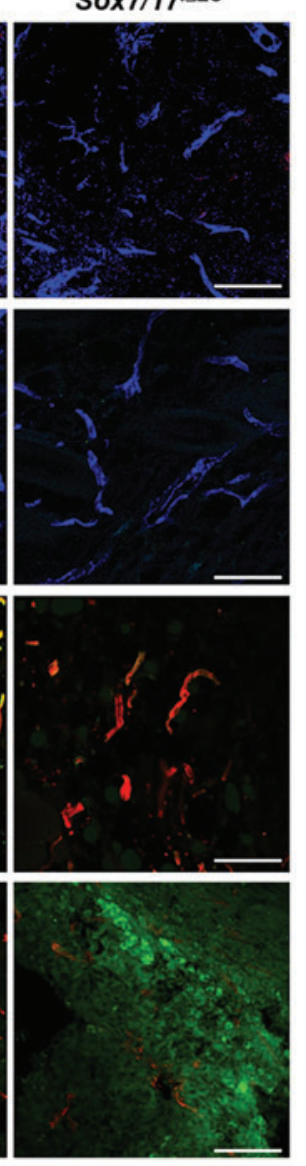

Scale bar: $100 \mu \mathrm{m}$
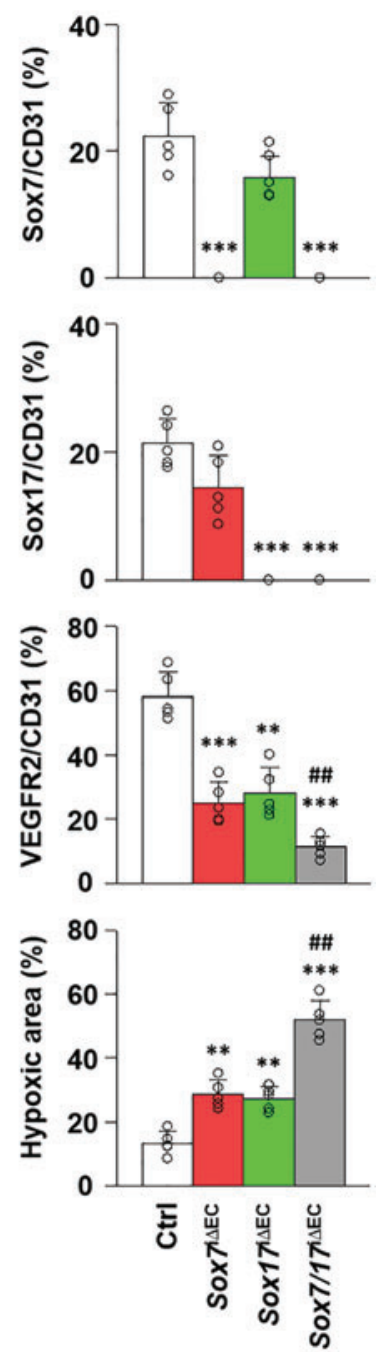

Figure 3. Sox7 or Sox 17 deletions did not suppress the expression of each other. (A) Sox7 immunostaining and its quantification in wound vessels. (B) Sox17 immunostaining and its quantification in wound vessels. (C) VEGFR2 immunostaining and its quantification in wound vessels. (D) Pimonidazole adduct (hypoxyprobe) and quantification of the hypoxic area. Values are presented as mean \pm SD $(n=5) .{ }^{* *} p<0.01 ;{ }^{* * *} p<0.001 ;{ }^{\# \#} p<0.05$ versus Sox7-KO. VEGFR, vascular endothelial growth factor receptor. Color images are available online.

or Sox17-deficient mice (Fig. 3D). In the wound tissue of Sox7/Sox 17 double-deficient mice, hypoxia exceeded $50 \%$, showing significantly increased levels in comparison with control, Sox7-, or Sox17deficient mice (Fig. 3D). These results correlated with the findings that Sox 7 and/or Sox 17 deletions reduce vascularity in the wound tissue, showing that the wound microenvironment is consequently affected.

\section{DII4 blockade promotes wound healing in Sox7-deficient mice}

During developmental angiogenesis, an antiDll4 antibody upregulates endothelial Sox17 and induces hyper-sprouting and the formation of a hyperdense vascular network. ${ }^{10}$ As Sox 7 deletion did not affect Sox17 expression, Dll4 blockade was conducted in Sox7-deficient mice to investi- gate whether Sox17 expression, angiogenesis, and wound healing could be enhanced. Concurrent with previous experiments, EC-specific Sox 7 deletion delayed wound healing in comparison with the control group (Fig. 4A, B). In contrast, Dll4 blockade (with the Sox7 deletion) reduced the wound size, similar to that observed in the control group, and nearly complete wound healing was observed on postoperative day 10 (Fig. 4A, B). These data indicate that Dll4 blockade improves nonhealing wounds induced by Sox7 deletion.

\section{DII4 blockade induces Sox17 expression and functional angiogenesis in Sox7-deficient mice}

To understand how Dll4 blockade restores delayed wound healing in Sox7-deficient mice, im- 

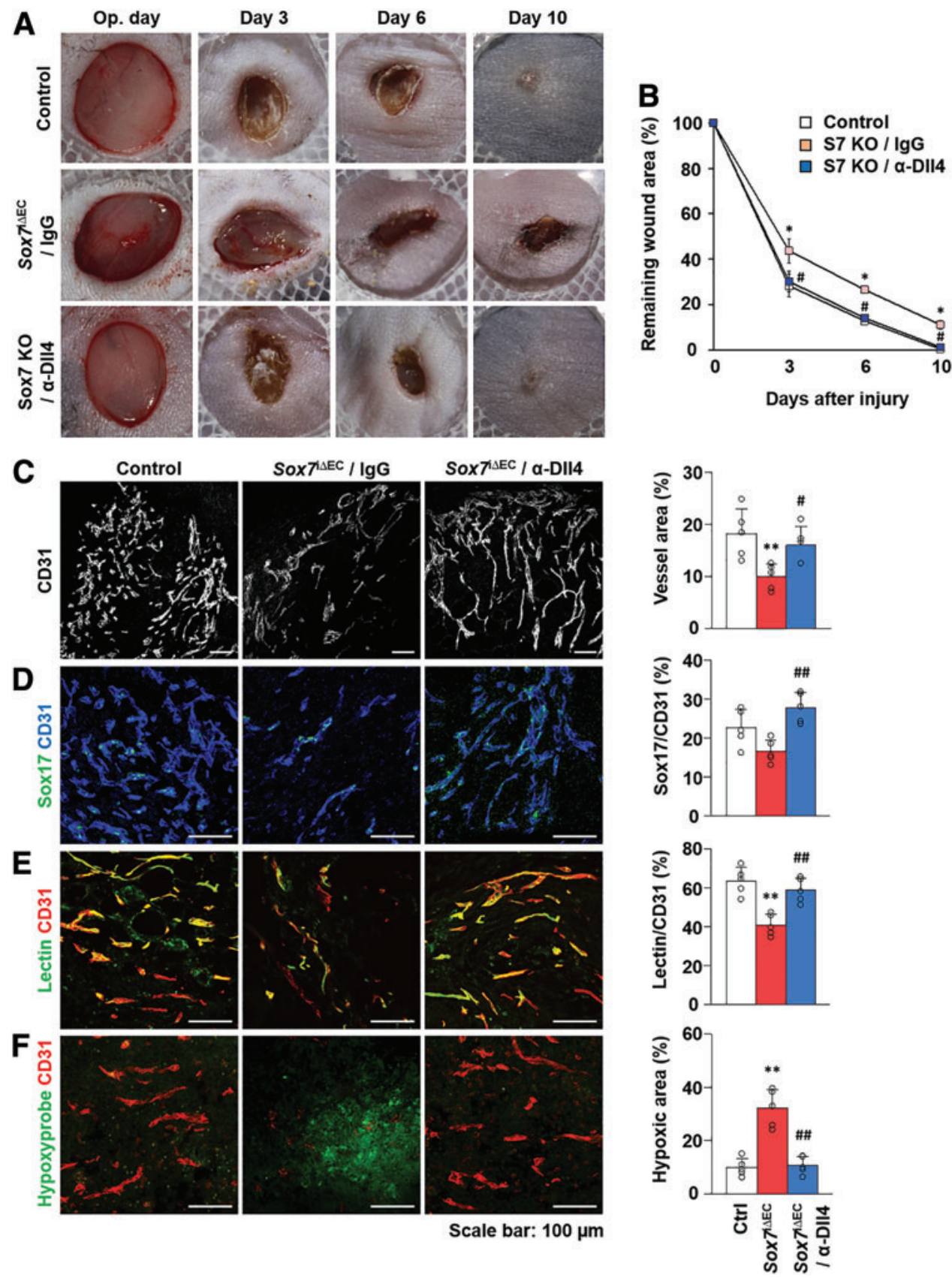

Figure 4. Treatment with anti-DII4 antibody accelerates wound healing in nonhealing wounds of Sox7-deficient mice. (A) Representative images of wounds at different time points. (B) Quantification of the wound area. (C) CD31-positive vessels and their quantification in wound tissues. (D) Sox17 immunostaining and its quantification in wound vessels. (E) Lectin immunostaining and quantification of vascular perfusion. (F) Pimonidazole adduct (hypoxyprobe) and quantification of the hypoxic area. Values are presented as mean \pm SD $(n=5)$. ${ }^{*} p<0.05 ;{ }^{* *} p<0.01$; ${ }^{\#} p<0.05$ versus Sox7-K0; ${ }^{\# \#} p<0.05$ versus Sox7-K0. Color images are available online.

munofluorescence analyses of vascularity, Sox17 expression, lectin perfusion, and hypoxia assessment in wound tissue were performed. Vascular density decreased in Sox7-deficient mice in comparison with control mice, whereas it was recovered in Sox 7 deletion mice treated with the anti-Dll4 antibody to a level similar to that in control mice (Fig. 4C). Sox17 was upregulated through Dll4 blockade in Sox7-deficient mice (Fig. 4D). Treatment with anti-Dll4 antibody improved vascular perfusion in Sox7-deficient mice and increased vascularity (Fig. 4E). Furthermore, tissue hypoxia was decreased through Dll4 blockade in Sox7-deficient mice (Fig. 4F). These results show that Dll4 inhibition upregulated endothelial Sox17 and functional angio- 
genesis in the nonhealing wounds induced through Sox7 deletion, thereby promoting wound healing.

\section{Wound healing was improved through DII4 blockade in $d b / d b$ diabetic mice}

Considering the functional restoration of wound angiogenesis through Dll4 blockade in Sox7deficient mice, we investigated whether treatment with anti-Dll4 antibody could accelerate delayed wound healing in $d b / d b$ diabetic mice. The nonhealing phenotype of chronic diabetic wounds is closely associated with impaired wound angiogenesis. ${ }^{14}$ After the generation of full-thickness skin defects in $d b / d b$ mice, anti-Dll4 antibody $(5 \mathrm{mg} / \mathrm{kg})$ was intraperitoneally administered at 7,10 , and 13 days after surgery. Wound healing was delayed in control $d b / d b$ mice to $>2.5$ weeks after surgery (Fig. 5A, B). Dll4 blockade improved wound healing and vascularity, upregulated Sox17, and enhanced lectin perfusion (Fig. 5B, C). These results confirm vasculogenic restorative effects through Dll4 blockade in the diabetic wound healing model.

\section{DISCUSSION}

The SoxF group, a subfamily of the Sry-related high-mobility group box (Sox) transcription factor family, consists of Sox7, Sox17, and Sox $18 .{ }^{15}$ Sox18 plays a crucial role in the development of the lymphatic vasculature. ${ }^{16}$ Sox7 and Sox17 are closely associated various types of angiogenesis. ${ }^{8-11,13,17}$ This is the first report of the proangiogenic role of
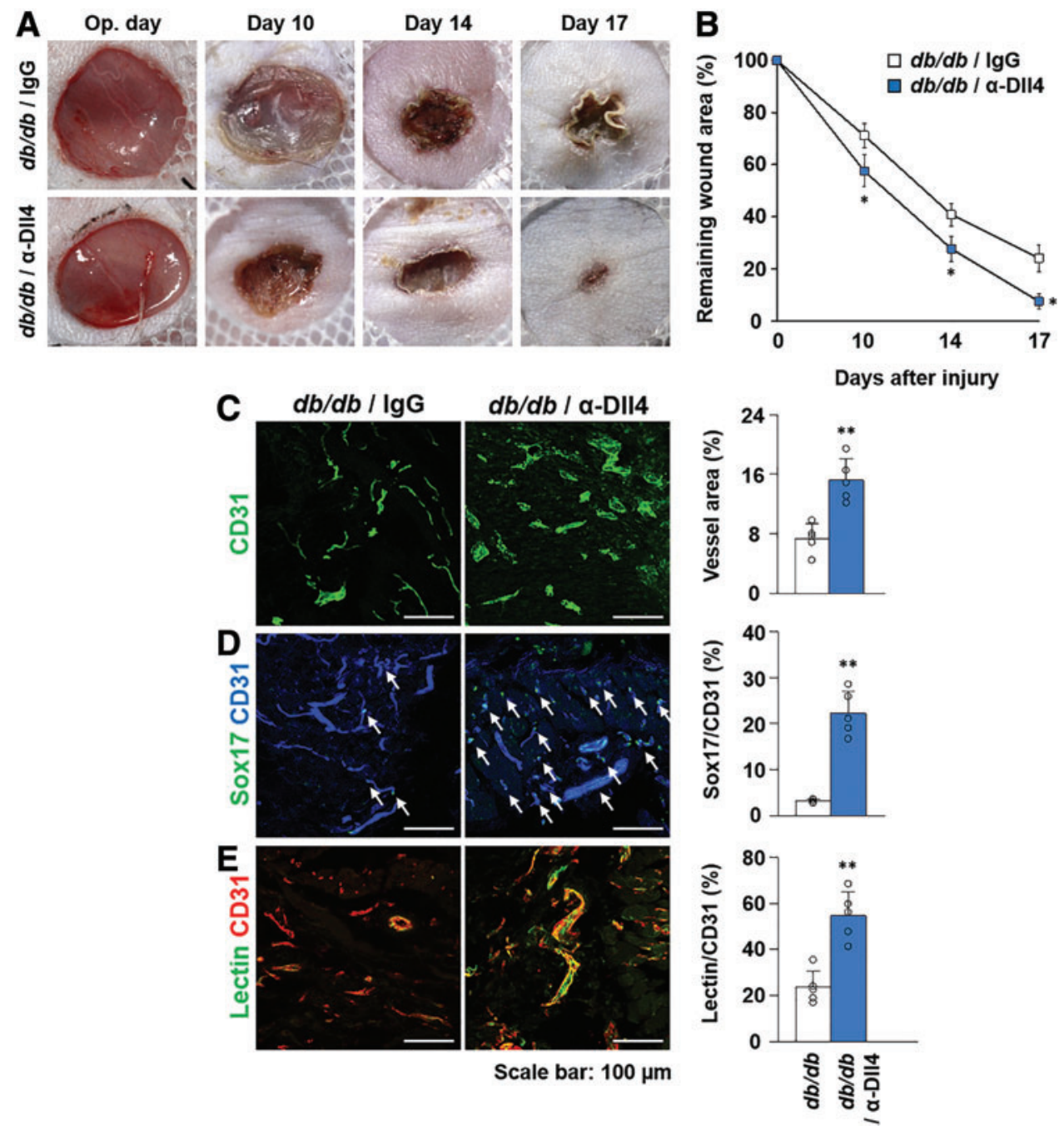

Figure 5. Treatment with anti-DII4 antibody promotes wound healing in $d b / d b$ diabetic mice. (A) Representative images of wounds at different timepoints. (B) Quantification of the wound area. (C) CD31-positive vessels and their quantification in wound tissues. (D) Sox17 immunostaining (white arrows) and its quantification in wound vessels. (E) Lectin immunostaining and quantification of vascular perfusion. Values are presented as the mean $\pm S D(n=5)$. ${ }^{*} p<0.05$; ${ }^{* *} p<0.01$. Color images are available online. 
Sox7 and Sox17 in wound healing. The present results show that these two transcription factors are upregulated in ECs during wound angiogenesis. EC-specific Sox7 and/or Sox17 deletion was possible by breeding the $C d h 5(\mathrm{BAC})-\mathrm{CreER}^{\mathrm{T} 2}$ driver line with $\operatorname{Sox} 7^{\mathrm{f} / \mathrm{fl}}$ and/or Sox $17^{\mathrm{f} / \mathrm{fl}}$ mice. Moreover, wound healing was delayed, and angiogenesis was reduced in Sox7- and Sox17-deficient mice. Upon blocking Dll4, which negatively regulates Sox17, this transcription factor was upregulated in Sox7-deficient mice and the delay in wound healing was rescued by the restoration of angiogenesis (Fig. 6).

Sox7 or Sox17 deletions did not alter the expression of the other molecules during wound healing, which constitutes an unexpected finding herein. We previously reported that Sox17 negatively regulates Sox7 expression in glioma angiogenesis. ${ }^{9}$ This nonredundant role of Sox7 and Sox17 in wound angiogenesis is similar to that in developmental angiogenesis. Sox7 and Sox17 expression did not depend on each other in the postnatal retinal vessels. ${ }^{8}$ Both developmental and wound angiogenesis are physiological phenomena; however, tumor angiogenesis is pathologic and Sox7 and Sox17 expression was heterogeneous in tumor vessels. This might be one reason for their different cooperation modes.

Several nonhealing wound models have been previously described. The most representative are diabetic chronic wounds. ${ }^{18,19}$ Furthermore, a wound model of chronic pressure and nonhealing wound models receiving radiation or chemotherapy have been reported. ${ }^{20-23}$ However, no study has reported a model of delayed wound healing due to suppressed angiogenesis in the wound tissue. Moreover, In fact, impaired angiogenesis was observed in a considerable number of patient tissues in which nonhealing wounds are found. Thus, Sox7- or Sox17-deficient mice can be used as a model of nonhealing wounds through impaired angiogenesis.

Sox 7 and Sox 17 are upregulated in angiogenic vessels of wound tissues and promoted wound angiogenesis. Hence, their expression can be considered an angiogenic marker to assess the extent to which angiogenesis occurs in a specific tissue. When histological analysis was performed with wound tissues, sole examination of vascular morphology can be problematic in determining whether the corresponding blood vessel is undergoing neovascularization or not. In contrast, immunostaining for Sox7 and Sox17 would help determine the occurrence of active angiogenesis in blood vessels. Thus, Sox7 and Sox17 levels can be considered diagnostic markers of nonhealing wounds occurring because of impaired angiogenesis.

The role of the Dll4-Notch signaling pathway in wound healing has been reported in several studies. Chigurupati et al. reported that wound healing is delayed when the Notch pathway is

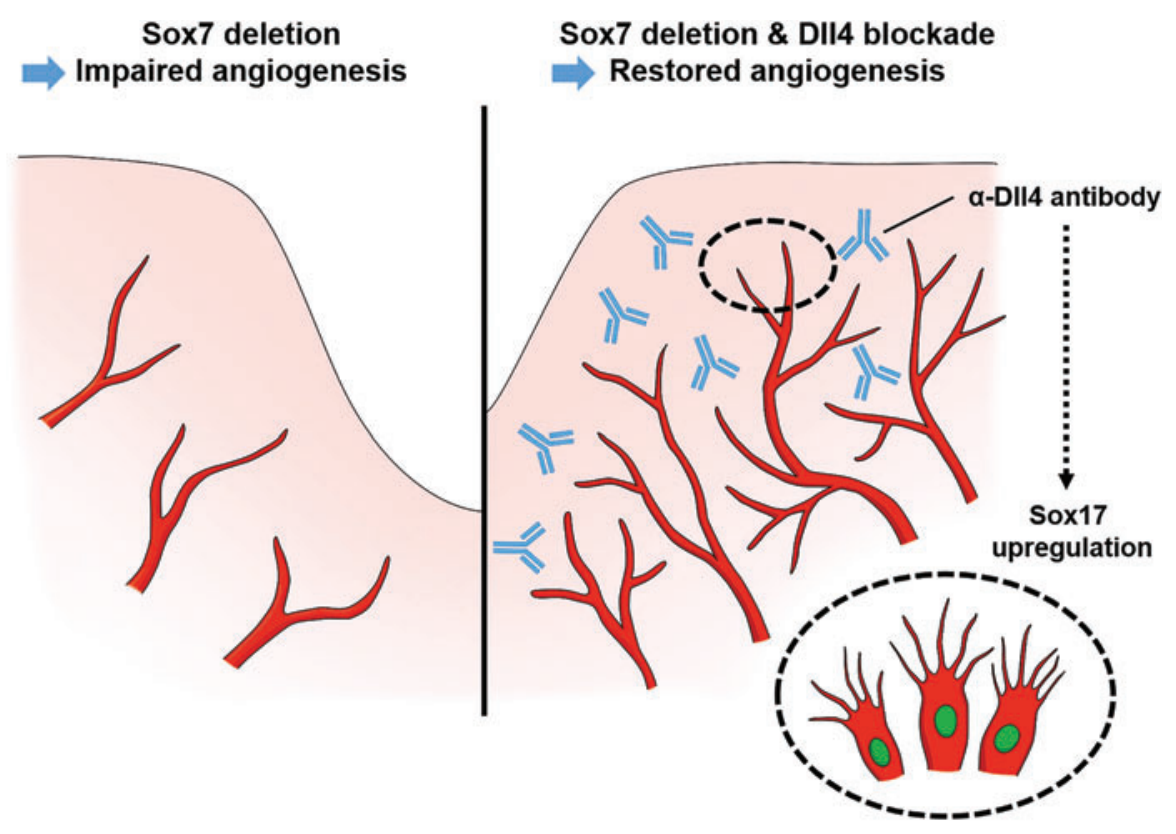

Figure 6. Diagram depicting restored angiogenesis and wound healing by DII4 blockade induced upregulation of endothelial Sox 17 expression in Sox7deficient mice. Color images are available online. 
inhibited; however, it was enhanced upon treatment with the activating Jagged-1 peptide. ${ }^{24}$ Trindade et al. reported that low-dosage Dll4 inhibition induced functional angiogenesis and consequently promoted wound healing. ${ }^{25}$ Together, the effect of Dll4-Notch inhibition on normal wound healing is controversial. However, we applied Dll4 blockade to nonhealing wounds with impaired angiogenesis. Inhibition of the Dll4Notch pathway leads to excessive angiogenesis and vascular leakage in physiological developmental angiogenesis and tumor angiogenesis. ${ }^{26,27}$ Hence, we hypothesized that this proangiogenic aspect of Dll4 blockade serves as a therapeutic agent especially in tissues lacking angiogenesis.

Our study has the following limitations. First, there is a lack of understanding of the direct molecular regulation of Sox17 by Dll4 during wound angiogenesis. Further comprehensive analysis is needed to determine the role of Dll4 in Sox17 regulation in nonhealing wounds with impaired angiogenesis. Second, our study primarily focused on the vascular phenotype in the wound model, thereby neglecting the effect of inflammatory cells playing a significant role in wound healing. Third, our study had a relatively short period $(<4$ weeks) for the wound model, which lacks the evaluation of the effect of Dll4 blockade in the chronic wound model with longer term follow-up. Finally, no clinical data were evaluated herein. Future studies on the treatment of human wounds with anti-Dll4 antibody are required to determine the efficacy of this novel treatment approach. Despite several limitations, to our knowledge, this study describes a significant and clinically relevant wound model, which is the first, to our knowledge, to suggest that Dll4 blockade potentially contributes to wound healing in patients with nonhealing wounds through impaired angiogenesis.

In conclusion, this study revealed the proangiogenic role of the transcription factors Sox7 and Sox17 in wound angiogenesis. Furthermore, a nonhealing wound model based on Sox7 and/or Sox17 deletion and a novel method for treating these lesions by targeting the Notch-Sox17 axis is suggested herein. This study potentially serves as a translational study to improve the treatment of chronic and nonhealing wounds caused by impaired angiogenesis.

\section{INNOVATION}

It remains necessary to develop a novel strategy to improve wound healing, particularly with respect to the treatment of nonhealing wounds. This study revealed the proangiogenic role of Sox 7 and Sox17 in wound healing. Furthermore, a novel nonhealing wound model based on $S o x 7$ and/or Sox17 deletion is suggested herein. Dll4 blockade upregulated endothelial Sox17 and angiogenesis in the nonhealing wound induced by Sox7 deletion and the diabetic wound model.

\section{ACKNOWLEDGMENTS AND FUNDING SOURCES}

This study was supported by National Research Foundation of Korea (grants NRF-2018 R1C1B6004618, 2019M3E5D1A02068102, and 2015R1A5A2009124 to I.-K.K. and 2018R1A2A1 A05022672 and Brain Research Program 2016 M3C7A1913844 to I.K.).

\section{AUTHOR DISCLOSURE AND GHOSTWRITING}

The authors declare no conflict of interest and ghostwriting services were not used.

\section{ABOUT THE AUTHORS}

Il-Kug Kim, MD, PhD, is a Professor of Plastic and Reconstructive Surgery at Yeungnam University College of Medicine. Hak Chang, MD, PhD, is a Professor of Plastic and Reconstructive Surgery at Seoul National University College of Medicine. Injune Kim, PhD, is a Professor of Graduate School of Medical Science and Engineering at the Korea Advanced Institute of Science and Technology. Jee Myung Yang, MD, is a graduate student in Professor Injune Kim's Lab at Korea Advanced Institute of Science and Technology. Junghwa Ryu, PhD, is a postdoctoral fellow in Professor Il-Kug Kim's Lab at Yeungnam University College of Medicine. 


\section{REFERENCES}

1. Risau W. Mechanisms of angiogenesis. Nature 1997;386:671.

2. Tonnesen $M G$, Feng $X$, Clark RA. Angiogenesis in wound healing. In J Invest Derm Smyp P 2000;5: 40-46.

3. Arnold F, West DC. Angiogenesis in wound healing. Pharmacol Ther 1991;52:407-422.

4. Echtermeyer F, Streit M, Wilcox-Adelman S, et al. Delayed wound repair and impaired angiogenesis in mice lacking syndecan-4. J Clin Invest 2001; 107:R9-R14.

5. Falanga V. Wound healing and its impairment in the diabetic foot. Lancet 2005;366:1736-1743.

6. Demidova-Rice TN, Durham JT, Herman IM. Wound healing angiogenesis: innovations and challenges in acute and chronic wound healing. Adv Wound Care 2012;1:17-22.

7. Li J, Zhang YP, Kirsner RS. Angiogenesis in wound repair: angiogenic growth factors and the extracelIular matrix. Microsc Res Tech 2003;60:107-114.

8. Kim K, Kim I-K, Yang JM, et al. SoxF transcription factors are positive feedback regulators of VEGF signaling. Circ Res 2016;119:839-852.

9. Kim I-K, Kim K, Lee E, et al. Sox7 promotes highgrade glioma by increasing VEGFR2-mediated vascular abnormality. J Exp Med 2018;215:963-983.

10. Lee S-H, Lee S, Yang H, et al. The Notch pathway targets proangiogenic regulator Sox17 to restrict angiogenesis. Circ Res 2014;115:215-226.

11. Yang $H$, Lee $S$, Lee $S$, et al. Sox17 promotes tumor angiogenesis and destabilizes tumor vessels in mice. J Clin Invest 2013;123:418-431.

12. Lobov I, Renard R, Papadopoulos N, et al. Deltalike ligand 4 (DII4) is induced by VEGF as a neg- ative regulator of angiogenic sprouting. Proc Natl Acad Sci U S A 2007;104:3219-3224.

13. Zhou Y, Williams J, Smallwood PM, Nathans J. Sox7, Sox17, and Sox18 cooperatively regulate vascular development in the mouse retina. PLoS One 2015;10:e0143650.

14. Okonkwo UA, DiPietro LA. Diabetes and wound angiogenesis. Int J Mol Sci 2017;18:1419.

15. Wei L, Yang C, Tao W, Wang D. Genome-wide identification and transcriptome-based expression profiling of the Sox gene family in the nile tilapia (Oreochromis niloticus). Int J Mol Sci 2016;17:270.

16. Francois M, Caprini A, Hosking B, et al. Sox18 induces development of the lymphatic vasculature in mice. Nature 2008:456:643-647.

17. Nakajima-Takagi $Y$, Osawa $M$, Oshima $M$, et al. Role of SOX17 in hematopoietic development from human embryonic stem cells. Blood 2013; 121:447-458.

18. Yang P, Pei Q, Yu T, et al. Compromised wound healing in ischemic type 2 diabetic rats. PLoS One 2016;11:e0152068.

19. Broadley K, Aquino A, Hicks B, et al. The diabetic rat as an impaired wound healing model: stimulatory effects of transforming growth factor-beta and basic fibroblast growth factor. Biotechnol Ther 1989;1:55-68.

20. Peirce SM, Skalak TC, Rodeheaver GT. Ischemiareperfusion injury in chronic pressure ulcer formation: a skin model in the rat. Wound Repair Regen 2000;8:68-76.

21. Wassermann E, Van Griensven M, Gstaltner K, Oehlinger W, Schrei K, Redl H. A chronic pressure ulcer model in the nude mouse. Wound Repair Regen 2009;17:480-484.
22. Cromack DT, Porras-Reyes B, Purdy JA, Pierce GF, Mustoe TA. Acceleration of tissue repair by transforming growth factor $\beta 1$ : identification of in vivo mechanism of action with radiotherapyinduced specific healing deficits. Surgery 1993; 113:36-42.

23. Curtsinger L, Pietsch J, Brown G, Ackerman D, Polk JH, Schultz G. Reversal of Adriamycin-impaired wound healing by transforming growth factor-beta. Surg Gynecol Obstet 1989;168:517-522.

24. Chigurupati S, Arumugam TV, Son TG, et al. Involvement of notch signaling in wound healing. PLoS One 2007;2:e1167.

25. Trindade A, Djokovic D, Gigante J, et al. Lowdosage inhibition of DII4 signaling promotes wound healing by inducing functional neoangiogenesis. PLoS One 2012;7:e29863.

26. Hellström M, Phng L-K, Hofmann JJ, et al. DII4 signalling through Notch1 regulates formation of tip cells during angiogenesis. Nature 2007;445: 776.

27. Noguera-Troise I, Daly C, Papadopoulos NJ, et al. Blockade of DII4 inhibits tumour growth by promoting non-productive angiogenesis. Novartis Found Symp 2007;283:106-120.

\section{Abbreviations and Acronyms}

$\mathrm{ECs}=$ endothelial cells

FITC $=$ fluorescein isothiocyanate

$\mathrm{GFP}=$ green fluorescent protein

$\mathrm{PBS}=$ phosphate-buffered saline

$\mathrm{SD}=$ standard deviation

$\mathrm{VEGF}=$ vascular endothelial growth factor

VEGFR $=$ vascular endothelial growth factor receptor 